\title{
Inter-reaction and site-specific diffusion of hydrogen defects in natural orthopyroxene at high temperatures
}

\author{
YAN YANG $^{1 *}$; QUNKE XIA ${ }^{1}$
}

${ }^{1}$ Key Laboratory of Geoscience Big Data and Deep Resource of Zhejiang Province, School of Earth Sciences, Zhejiang University (*Correspondence: yanyang2005@zju.edu.cn)

As the second abundant constituent mineral of the upper mantle, aluminous orthopyroxene could be a more important host for water than olivine in the upper mantle. Although plenty of studies have explored hydrogen defects in orthopyroxene, it is still difficult to explain the discrepancies between hydrogen defects in the synthetic and natural orthopyroxenes. One of the reasons could be that hydrogen defects in natural samples from the mantle may experience complex modifications at high temperatures in the deep Earth. Indeed, several studies have revealed some interesting variations of hydrogen defects in the natural orthopyroxene from the mantle. For example, some researchers observed the typical hydrogen loss profiles from the core to rim [1], while obvious gain of some hydrogen defects from the core towards rim were reported [2].

To clarify what happens to hydrogen defects in orthopyroxene at high temperatures, here, we carry out investigations on hydrogen defects in three natural orthopyroxene samples at high temperatures to $1000{ }^{\circ} \mathrm{C}$. The results show that some new hydrogen defects appear accompanied by dehydrogenation of other hydrogen defects, indicating temperature-induced inter-reaction of hydrogen defects. On the other hand, the three samples all show sitespecific hydrogen diffusivities, with the fastest diffusivities at $3420 \mathrm{~cm}^{-1}$, and the slowest at $3560 \mathrm{~cm}^{-1}$. But the difference is not more than one order of magnitude. These results are necessary for interpreting hydrogen defects in natural orthopyroxene and evaluating the extent to which orthopyroxene from the mantle can preserve its original hydrogen signature.

[1] Tian et al. (2017) Geology 45, 87-90. [2] Tollan \& Hermann (2019) Nature Geosci. 12, 667-671. 